\title{
Announcement of the 2019 JCS Meeting Activities (2)
}

\author{
Toyoaki Murohara, MD, PhD
}

Dear Colleagues,

As the Editor-in-Chief of the Circulation Journal, the official journal of the Japanese Circulation Society (JCS), I am pleased to further report the JCS 2019 Meeting Activities.

\section{JCS 2019 Activities}

The JCS 2019 Annual Meeting has been held during March 29-31 in Yokohama, Japan. The following winners of the Young Investigator's Award (YIA), the YIA for International Students and the Co-medical Award have been selected and were awarded during the Meeting. These awards should promote their careers.

\section{Young Investigator's Award $\left(36^{\text {th }}\right)$}

\section{Clinical Research}

[First Place]

Akira Suda, MD

(Department of Cardiovascular Medicine, Tohoku University Graduate School of Medicine)

"Comprehensive Evaluation of Coronary Functional Abnormalities in Patients With Chest Pain and Unobstructive Coronary Artery Disease"

[Second Place] (in alphabetical order)

Shinichi Goto, MD, PhD

(Department of Cardiology, Keio University School of Medicine)

"Artificial Intelligence With Recurrent Neural Network to Predict the Need for Urgent Coronary Revascularization From 12-Leads Electrocardiography in Patients With Chest Discomfort"

Naotsugu Iwakami, MD, MPH

(Department of Research Promotion and Management, National Cerebral and Cardiovascular Center)

"Optimal Sampling in Derivation Studies Determines Performance of Heart Failure Prognostic Models"

Takuya Nagata, MD

(Department of Cardiovascular Medicine, Graduate School of Medical Sciences, Kyushu University)

"N-Terminal Pro-B-Type Natriuretic Peptide and Risk of Dementia in A General Japanese Elderly Population: The Hisayama Study"

\section{Basic Research \\ [First Place]}

Ryo Kurosawa, $\mathrm{PhD}$

(Department of Cardiovascular Medicine, Tohoku University Graduate School of Medicine)

"Identification of Celastramycin as A Novel Therapeutic Agent for Pulmonary Arterial Hypertension: Highthroughput Screening of 5,562 Compounds"

J-STAGE Advance Publication released online April 16, 2019

Department of Cardiology, Nagoya University Graduate School of Medicine, Nagoya, Japan

Mailing address: Toyoaki Murohara, MD, PhD, Department of Cardiology, Nagoya University Graduate School of Medicine, 65 Tsurumai, Showa-ku, Nagoya 466-8550, Japan. E-mail: murohara@med.nagoya-u.ac.jp

ISSN-1346-9843 All rights are reserved to the Japanese Circulation Society. For permissions, please e-mail: cj@j-circ.or.jp 
[Second Place] (in alphabetical order)

Hidenori Kojima, MD

(Cardiology Department, Keio University Hospital)

"Mef2c Directly and Indirectly Amplifies Cardiogenic Program Cooperating With P300 in Direct Cardiac Reprogramming"

Junichi Sugita, MD, PhD

(Cardiovascular Department, The University of Tokyo)

"Amphiregulin Derived From Cardiac Macrophages Prevents Lethal Arrhythmias"

Hidenori Tani, MD

(Department of Cardiology, Keio University School of Medicine)

"In Vivo Direct Reprogramming of Fibroblasts Into Cardiomyocytes Improves Cardiac Function in Chronic Heart Failure"

\section{Young Investigator's Award for International Students (17 $\left.{ }^{\text {th }}\right)$ \\ [First Place]}

Hilman Zulkifli Amin, MD (Indonesia)

(Division of Cardiovascular Medicine, Department of Internal Medicine, Kobe University Graduate School of Medicine, Kobe)

"Overexpression of Cytotoxic T-Lymphocyte-Associated Antigen-4 (CTLA-4) Protects Against Abdominal Aortic Aneurysm Formation in Mice"

[Second Place] (in alphabetical order)

Sari Nurmila (Indonesia)

(Graduated School of Integrated Sciences and Technology, Shizuoka University, Shizuoka)

"Cocoa Bean Polyphenols Inhibit Cardiac Hypertrophy and Dysfunction in Pressure Overload-Induced Heart Failure Model"

Zhongyue $\mathrm{Pu}, \mathrm{MD}$ (China)

(Department of Cardiology, Nagoya University Graduate School of Medicine, Nagoya)

"Lymphangiogenesis Contributes the Ischemia Induced Angiogenesis in HLI via Clearance of Inflammation"

Vijayakumar Sukumaran, $\mathrm{PhD}$ (Indonesia)

(National Cerebral and Cardiovascular Center Research Institute, Suita)

"Liraglutide Treatment Improves the Coronary Microcirculation in Zucker Obese Rats on High-Salt Diet"

Fikri Taufiq, MD (Indonesia)

(Division of Regenerative Medicine and Therapeutics, Tottori University, Yonago)

"Uric Acid-Induced Increases of Protein and Channel Activity of Atrial Kv1.5 via Akt-HSF1-Hsp70 Axis"

\section{Co-medical Award (9th)}

\section{Inspection and Treatment Session}

\section{[First Place]}

Naoya Kakutani, MSc, BSc

(Department of Cardiovascular Medicine, Faculty of Medicine and Graduate School of Medicine, Hokkaido University) "Development and Implementation of Progressive Mobilization Program for Patients With Acute Heart Failure"

[Second Place] (in alphabetical order)

Toshihiro Hayashi

(Department of Radiology, University of Tokyo Hospital)

"Multicenter Survey on Radiation Dose of Cardiac Intervention"

Hitoshi Ono

(Clinical Engineering Center, Chiba University Hospital)

"Is it Possible to Predict Inappropriate Therapy of Implantable Defibrillator Due to Atrial Fibrillation?" 
[Third Place] (in alphabetical order)

Miki Akiyama, Bpharm

(Department of Pharmacy, Saku Central Hospital Advanced Care Center)

"The Role of Pharmacists in Supporting Home Dobutamine Therapy for Inotrope-Dependent Patients With End-Stage Heart Failure"

Soichiro Tominaga

(Department of Clinical Laboratory, Kochi Medical School Hospital)

"Detection for the Prediction of Heart Failure Events Using Ventricular Late Potentials in Patients With Hypertrophic Cardiomyopathy"

Yuta Torii, Master of Science

(Ultrasound Examination Center, Tokushima University Hospital)

"Effect of Calf Sleeve on Leg Edema: Evaluation Using Superficial Ultrasonography"

\section{Nursing, Medicine and Rehabilitation Session}

\section{[First Place]}

Takuji Adachi, BSc, PhD

(Department of Health Sciences, Nagoya University Graduate School of Medicine)

"Self Efficacy for Physical Activity and Depresson Are Associated With Appetite Loss in Frail Patients With Heart Failure: A Multicenter Cohort Study (FLAGSHIP)"

[Second Place] (in alphabetical order)

Takatoshi Iwasaki, MS

(Minamino Cardiovascular Hospital)

"Relation Between Peak VO2 of Heart Failure and Epicardial Adipose Tissue in Cardiac Rehabilitation"

Kiyonori Kobayashi, MSc

(Department of Rehabilitation, Nagoya University Hospital)

"A Relationship of Recovery State From Skeletal Muscle Weakness During Perioperative Left Ventricular Assist Device Surgery"

[Third Place] (in alphabetical order)

Yoshitaka Naito

(Department of Rehabilitation, The Sakakibara Heart Institute of Okayama)

"Contributing Factors for Readmission After Transcatheter Aortic Valve Implantation"

Daisuke Yamaguchi, MSN

(Division of Nursing, School of Health Sciences, Shinshu University)

"Depression Coping Strategies in Patients With Coronary Artery Disease and Type D Personality"

Junichi Yokota, MS

(Department of Internal Medicine and Rehabilitation Science, Tohoku University Graduate School of Medicine)

"Dysphagia Affects Short-Term Outcomes in Patients With Acute Exacerbation of Heart Failure"

Toyoaki Murohara, MD, $\mathrm{PhD}$

Editor-in-Chief

Circulation Journal 